\title{
Research on obstacle recognition and distance calculation based on the structure of the road
}

\author{
Zou Zi-ming ${ }^{1, a}$,Li Hai-Rong ${ }^{1, b}$,Liu Xiao-yang ${ }^{1, c}$ \\ ${ }^{1}$ College of Mechanical and Control Engineering, Guilin University of Technology, Guilin 541004, \\ Guangxi, China \\ aemail: zzm567567@163.com bemail:1152203173@qq.com cemail:595780975@qq.com
}

Key words: obstacle recognition; distance calculation; the structure of road.

Abstract. Based on Machine Vision, the paper research a method to detect obstacles and calculate the distance between the obstacle and moving vehicles. First it uses the shadow detection method to determine approximate location of the obstacle and adopt corner detection method to detect the obstacle contour information. Then, the minimum bounding rectangle method is used to accurately framed obstacles vehicles. Finally, based on the principle of the distance in the camera, the obstacle distance measurement formula is built. Experimental results show that the algorithm can accurately locate obstacles and measure the actual distance between the moving vehicle and the obstacles.

\section{Introduction}

Based on Machine Vision, the assistance driver systems can capture traffic information via CCD camera, use image processing techniques to analyze road conditions, detect obstacle in the front of the vehicle and calculate the distance between vehicle and obstacle to avoid traffic accident. Obstacle detection methods and the obstacle distance algorithm is studied in this article.

\section{Obstacle shadow detection}

In the grayscale image of the road, the gray values have a step of the transition between the shaded area of the vehicle and the other area of the road, which will determine the site of the vehicle obstacle $^{[1]}$. Algorithm is designed as follows.

Firstly, the road images captured by the CCD camera are color images, which need to transform into grayscale images via the weighted average method. Then we uses sobel operator to edge enhancement, the horizontal and vertical of nuclear core are shown in Fig.1. It can enhance the vehicle edge information and vehicle shadow information to improve the accuracy of detection.

$$
\Delta f_{*}(x, y)=\left[\begin{array}{ccc}
-1 & -2 & -1 \\
0 & 0 & 0 \\
1 & 2 & 1
\end{array}\right] \quad \Delta f_{i}(x, y)=\left[\begin{array}{ccc}
-1 & 0 & 1 \\
2 & 0 & 2 \\
-1 & 0 & 1
\end{array}\right]
$$

Fig. 1 the Sobel operator of horizontal direction and the vertical direction

Then, In the area between the left and right lane where the vehicle was, we get the sum of the gray value of each pixel in the row, and divide by the width between the left and right lane to get the mean gray value of each line, this value is called projection means $H(n)_{a v}$, as shown in Eq.1.

$$
H(\mathrm{n})_{\mathrm{av}}=\frac{1}{\mathrm{~b}(\mathrm{n})-\mathrm{b}(\mathrm{n})+1} \sum_{\mathrm{cob(n)}}^{\mathrm{b}(\mathrm{n})} \mathrm{g}(\mathrm{n}, \mathrm{c})
$$

Wherein, $b(n)_{l}$ and $b(n)_{r}$ respectively represent the pixel coordinate of left lane and the pixel coordinate of the right lane in the $\mathrm{n}$ row; $g(n, c)$ represents the gray value of a point of image pixel.

Finally, Eq. 2 is used for weighting the projection means $H(n)_{a v}$ to obtain two threshold values. The one $T(n)_{\text {tre }}$ is adaptive threshold of the image which is the enhanced image of sobel edge operator, and the other $T(n)_{\text {ori }}$ is an adaptive threshold of the original grayscale image. According to these two thresholds, the article can determine whether the presence of the vehicle shadow. 


$$
\begin{aligned}
& T(\mathrm{n})_{\text {ur }}=\alpha \cdot H(\mathrm{n})_{\mathrm{v}}, \\
& T(\mathrm{n})_{\text {oi }}=\beta \cdot H(\mathrm{n})_{\text {av }} .
\end{aligned}
$$

Wherein, $\alpha$ and $\beta$ are weighting coefficients. We set $\alpha=0.25, \beta=0.5$.

When the original gray value of a pixel is smaller than $T(n)_{o r i}$, and after enhancing by the sobel operator edge, it's gray value is greater than $T(n)_{t r e}$, which can be judged the pixel is shadow points of the vehicle. After detecting the shadow image information of the vehicle, we get the preliminary location information of the obstacle. But if the road surface has the shadow of other objects, such as street signs, the obstacle region might be wrong by shadow detection method. Therefore, the corner point method is used in the obstacle recognition to improve identification accuracy.

\section{Corner Detection}

The basic idea ${ }^{[2]}$ of the USAN corner detection algorithm as shown in Fig.2: The circle templates were placed at abcde position (as shown Fig.2(a)) in rectangular region. The difference of the pixel of the round template and the gray value of each pixel core is calculated, and compared it with the set threshold, the comparison function as shown in Eq.3.

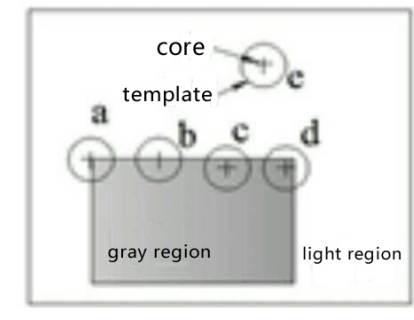

( a )

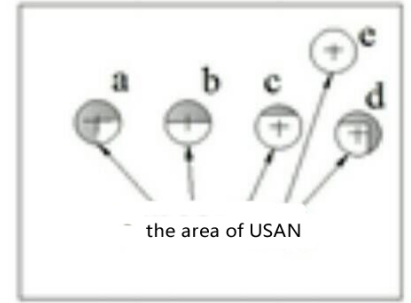

( b )

Fig.2 Corner Detection

$$
c\left(x, y, x_{0}, y_{0}\right)=\left\{\begin{array}{ll}
1 & \left|I(x, y)-I\left(x_{0}, y_{0}\right)\right| \leq T \\
0 & \mid I\left(x, y-I\left(x_{0}, y_{0}\right) \mid>T\right.
\end{array} .\right.
$$

$I\left(x_{0}, y_{0}\right)$ is the gray value of the core pixel in the circular template at the current position, $I(x, y)$ is the other pixel gray value in the circular template. $T$ is the gray difference threshold, which can distinguish between the threshold of the target and background. $T$ is generally ranges from 10-25. The value of $T$ needs to take into account the noise and the nature of characteristic points.

This paper selects circular template which contains 37 pixels as shown in Fig.3. The circle template is larger, corner points are more detected, the amount of computation is greater. We compare the gray value of each pixel with the core pixel in the template by using Eq. 3 to obtain the set of pixel which the gray value is closer to the pixel core. It is called Univalue Segment Assimilating Nucleus (USAN), the white area of Fig2.(b) is the USAN. The pixel that the gray value is closer to the pixel core is called similarities point, the sum of similarities point in circular template expressed the area of USAN, which is denoted by $n$. According to Eq.4, we get the maximum value of the USAN is 36 (pixel).

$$
\mathrm{n}_{\max } / 4=9(\text { pixel }) .
$$

According to the value of $n$, we can determine the core pixel is corner points or not be, we usually define a response function to divide the value of the response in the statistical process, the 
result of the response can determine the position of corner points. The response results is the larger, the likelihood of corner point is greater. In Fig.2(a), a, d can be considered as corner points. Response function is shown as Eq.5.

$$
R\left(\mathrm{x}_{0}, \mathrm{y}_{0}\right)=\left\{\begin{array}{cc}
\mathrm{g}-\mathrm{n}\left(\mathrm{x}_{0}, \mathrm{y}_{0}\right) & \mathrm{n}\left(\mathrm{x}_{0}, \mathrm{y}_{0}\right)<\mathrm{g} \\
0 & \mathrm{n}\left(\mathrm{x}_{0}, \mathrm{y}_{0}\right) \geq g
\end{array} .\right.
$$

\section{Morphological expansion dealing}

Corner detection has been able to show the approximate outline of obstacles but relatively empty. On the basis of the corner detection, the image can be morphological processed to make the area more obvious obstructions. The paper uses the expansion operator ${ }^{[3]}$.Algorithm steps are as follows.

We select the structural element that if is selected too large, the operation time is too long, contrary, or is selected too small, the effect is not obvious. This selection of $3 * 3$ structure element is shown in Fig.4, the origin point is local in the center position. the origin of the structural element $\mathrm{S}$ move to the pixel point in the image $\mathrm{F}$ which is denoted as $f(x, y)$, each pixel in the structural elements make "and" operation with the corresponding pixel which is covered by the structural elements in the original image. If both are 0 , in the original image, the pixel $f(x, y)$ is set as 0 . Otherwise, the pixel $f(x, y)$ is set as 1 .

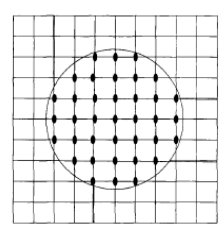

Fig.3 pixel circular template

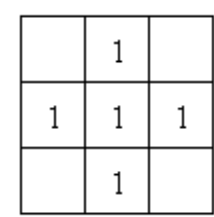

Fig. $43 * 3$ structure element

Finally, it constantly move structural elements $\mathrm{S}$, and make origin point of structural elements $\mathrm{S}$ cover over all the pixels in the original image.

\section{The minimum bounding rectangle to fix obstacles}

After identifying the approximate location and the outline of the obstacle vehicle, the minimum bounding rectangle method will be used to accurately frame the obstacle vehicle location, which contains all the information of the graph, and has the smallest area ${ }^{[4]}$. In this paper, the rotation method is used to calculate the minimum bounding rectangle of obstacle vehicle, the basic idea is:

Firstly, the centroid $B\left(x_{0}, y_{0}\right)$ of the obstacle vehicle area is calculated according to Eq. 6

$$
\mathrm{x}_{0}=\frac{\int_{A} \mathrm{xd} A}{A} \quad, \quad \mathrm{y}_{0}=\frac{\int_{A} \mathrm{yd} A}{A} .
$$

Where: A is planar graphics area. When the image processing calculates the centroid, a pixel will be seen as a dA, so the area of image is the number of pixels, the integral of the molecule in Eq. 6 is the sum of the pixel coordinates.

Secondly, the image area around the obstacle vehicle centroid rotates an intervals angle. A compromise between accuracy and real-time is selected, each rotation angle is set as $3^{\circ}$.Totals of angle of rotation cannot exceed $90^{\circ}$, respectively, the maximum and minimum coordinates of each barrier region in the x-axis, $\mathrm{y}$-axis is denoted as $x_{\min }, x_{\max }, y_{\min }, y_{\max }$, and according to the four points of a rectangular, the Eq.7 is used to calculate the area of bounding rectangle of obstacle vehicle.

$$
S=\left(\mathrm{x}_{\max }-x_{\min }\right) \times\left(y_{\max }-y_{\min }\right)
$$


Finally, comparing each rectangular area of external rotation, the smallest minimum bounding rectangle is obtained.

\section{The obstacle distance measurement}

In the reference ${ }^{[5]}$, the camera imaging model is given a detailed introduction, and then based on this, in the image, the left edge of the L position in the coordinate of the camera system is (XL, H, $\mathrm{L})$, and in the image the coordinates is $(\mathrm{xL}, \mathrm{y})$; the right edge coordinates in the camera coordinate system is $(\mathrm{XR}, \mathrm{H}, \mathrm{L})$, and in the image the coordinates is $(\mathrm{xR}, \mathrm{y})$, the focal length of the camera is $\mathrm{f}$, the installation of the camera height is $\mathrm{H}$. We has the Eq.8.

$$
L=\mathrm{f} \frac{X_{R}-X_{L}}{\mathrm{X}_{\mathrm{R}}-\mathrm{X}_{\mathrm{L}}}=\mathrm{f} \frac{W_{\text {Road }}}{W_{\text {inrd }}}
$$

Wherein, the $W_{\text {Road }}$ is a actual width of the lane, the $W_{\text {imrd }}$ is a width of the lane where the obstacle in on the image. Some explanation for the Eq.8:

(1) It is only suitable for the structure of the road which has the fixed width of the lane, for example , the highway roads.

(2) Wherein, $\mathrm{L}$ is the distance from the front obstacle to the imaging plane.

(3) Eq.8 was derived from a simplified model which uses a perspective transformation method, the simplified model requirements $\alpha \rightarrow 0, \beta \rightarrow 0$.

Further, we can obtain the Eq.10 through Eq.9 to calculate the distance.

$$
\begin{aligned}
& L=-\mathrm{f} H / y \\
& L=-\mathrm{f} d /\left|x_{2}-x_{1}\right| .
\end{aligned}
$$

Wherein the variable $y$ is the row coordinate of car bottom edge on the image, the variable $\mathrm{d}$ is the width of the car body, the variable $x_{1}$ is left edges of the car body on the image,the variable $x_{2}$ is right edges of the car body on the image. Eq.9 needed for the variable $y$ alone, and has great possibilities to be interference, the automobile bumps jitter will cause $y$ great changes in a short period of time, therefore, it is not available for the calculation of the formula $L$. However, calculating the distance $L$ by other more accurate method, it can be estimated the variable $y$ that it was the row coordinate of car bottom edge on the image in the absence of noise. Compared with the actual row coordinate y of car bottom edge on the image, it can be roughly deduced the camera parameters mutations caused by the automobile bumps jitter. Eq. 8 and Eq.10 are very similar in form, the desired variable is a horizontal coordinate of the image, when the vehicle bumps jitter, $x$ can reduce the error of the difference between the jitter caused in large part. The distance $L$ we can get by weighting the two values which is calculated according to Eq.11. It is shown as Eq.12.

$$
\begin{aligned}
& L_{1}=\mathrm{fd} /\left|x_{2}-x_{1}\right| \quad L_{2}=\frac{\mathrm{f} W_{\text {Road }}}{W_{\text {ind }}} . \\
& L=\alpha L_{1}+(1-\alpha) L_{2} \quad, \quad 0<\alpha<1 .
\end{aligned}
$$




\section{Experiment}

We design of algorithm using Matlab programming to process to the road image acquisition, the experimental results were shown in Fig.5.

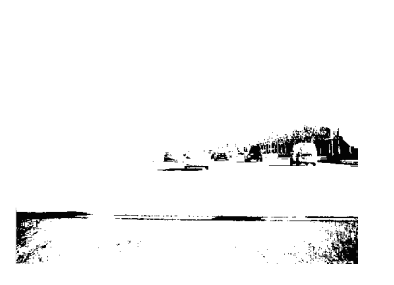

(a)

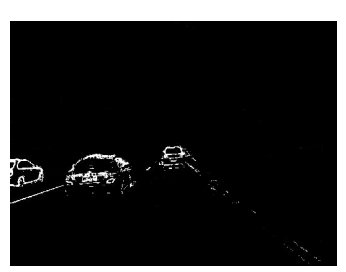

(b)

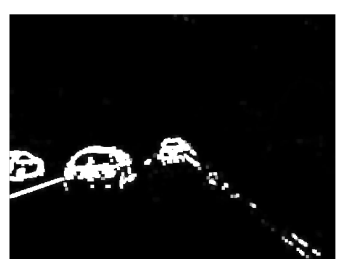

(c)

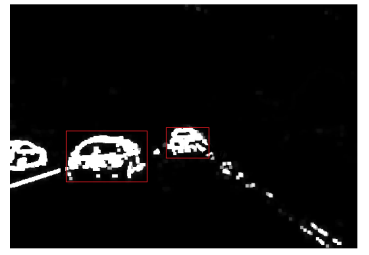

(d)

Fig.5 (a) shadow detection (b) corner detection (c) Physical Science expansion deal (d)minimum bounding rectangle

\section{Conclusions}

As can be seen from the experimental result, for the structure of the road, the design algorithm can eliminate the interference of trees and signs on both sides of the road, and can accurately identify the obstacles. The design algorithm can be more precise estimates of the distance between the obstacle and moving vehicles, it is able to meet the driver assistance systems on the implementation of the requirements, and has better robustness.

\section{Reference}

[1] Liu Huajun. A new method of generalized obstacle detection based on monocular vision [J]. Electronic journals, 2011.08:1793-1798

[2] Hou Mingliang . SUSAN corner detection algorithm improved stability study [J]. Computer and Modernization ,2010,10:75-77

[3] Yang Kun, Zeng Libo, Wang Diancheng. A fast algorithm for computing mathematical morphology corrosion expansion [J]. Computer Engineering and Applications , 2005, 34:54 56

[4] Lu Rong, Fan Yong, Chen Niannian, Wang Junbo. A fast algorithm for extracting minimum bounding rectangle of the target image [J]. Mianyang: School of Computer Science and Technology, Southwest University of Science and Technology Institute of Defense Technology, 2010, ( 21 ):178 -180

[5] Zhang Yingjin. Image Engineering-image processing and analysis. Beijing: Tsinghua University Press .1999

[6] Tang Gaoyou. Research road recognition and obstacle detection technology based on machine vision [D]. Heilongjiang: Chongqing University, 2005 